\title{
Large photonic band gaps in certain periodic and quasiperiodic networks in two and three dimensions
}

\author{
Sai-Kit Cheung, Tzu-Liang Chan, Zhao-Qing Zhang, and C. T. Chan \\ Department of Physics, Hong Kong University of Science and Technology, Clear Water Bay, Kowloon, Hong Kong, China \\ (Received 30 September 2003; revised manuscript received 12 April 2004; published 10 September 2004)
}

\begin{abstract}
The photonic band structures in certain two- and three-dimensional periodic networks made of onedimensional waveguides are studied by using the Floquet-Bloch theorem. We find that photonic band gaps exist only in those structures where the fundamental loop exhibits antiresonant transmission. This is also true for quasiperiodic networks in two and three dimensions, where the photonic band structures are calculated from the spectra of total transmission arising from a source inside the samples. In all the cases we have studied, it is also found that the gap positions in a network are dictated by the frequencies at which the antiresonance occurs.
\end{abstract}

DOI: 10.1103/PhysRevB.70.125104

PACS number(s): 42.70.Qs, 41.20.Jb

\section{INTRODUCTION}

In the last decade, significant effort has been devoted to the study of photonic band-gap (PBG) materials, in which the dielectric constant is periodic in space. ${ }^{1,2}$ The existence of a spectral gap in which electromagnetic waves cannot propagate in any direction offers the possibility to confine and control the propagation of electromagnetic waves. It can give rise to many interesting physical phenomena, as well as wide applications in several scientific and technical areas. For example, on the scientific side, it has been suggested that the Anderson localization, which is complicated by the $\mathrm{e}-\mathrm{e}$ interactions in an electronic system, is much easier to achieve inside the PBGs of a disordered photonic system. ${ }^{3}$ In practical applications, ultralow-threshold semiconductor lasers lie in the existence of robust microcavity modes in PBG systems, facilitated by the suppression of spontaneous emission of light. ${ }^{4}$ Besides periodic structures, PBG has also been found in certain quasiperiodic systems in two dimensions. ${ }^{5-8}$

Recently, a different kind of band gap structure for EM wave systems has been proposed. These are systems of networks made of one-dimensional waveguides, ${ }^{9}$ which are experimentally realizable, even in an undergraduate laboratory. In particular, by using coaxial cable as the $1 \mathrm{D}$ waveguide, a large photonic band gap has been found in a 3D network in the diamond structure, in which certain 1D waveguides have been replaced by resonant loops to produce strong scattering. ${ }^{9}$ In the presence of defect and randomness, both defect states and the Anderson localized states have also been observed inside the gap. Furthermore, network systems are also interesting for the study of many other potential physical problems such as PBG in fractals, ${ }^{11}$ and the wave localization in dimensions larger than three.

In this work, we have calculated the band structures of some basic lattice structures in two and three dimensions. We have also studied the photonic band structures in certain quasiperiodic networks in two and three dimensions by calculating the spectra of total transmission arising from a source inside the samples. In all the cases we have studied, it is found that the existence of large gaps in a network relies mainly on the presence of triangular loops in the fundamental building blocks of the lattice. Each loop is capable of producing antiresonances at certain frequencies with zero transmission. These frequencies coincide with the positions of the gaps found in the network. The outline of this paper is as follows: Sec. II describes the methods to calculate the band structures and the spectra of total transmission. The results of certain periodics are also presented. Section III presents the results of certain quasiperiodic networks, and Sec. IV gives the summary and discussions.

\section{PBGs IN PERIODIC NETWORKS}

A network consists of certain nodes connected by segments of 1D waveguides. The wave function at each node satisfies the following network equation: ${ }^{10}$

$$
-\varphi_{i} \sum_{j}\left[\cot \left(\omega l_{i j} / c\right)\right]+\sum_{j}\left[\frac{1}{\sin \left(\omega l_{i j} / c\right)}\right] \varphi_{j}=0,
$$

where $\varphi_{i}$ is the scalar wave function at node $i, l_{i j}$ is the length of the 1D waveguide between nodes $i$ and $j, \omega$ and $c$ are, respectively, the frequency and speed of the waves. The summation of $j$ is over all the nodes linked to node $i$. In the case of coaxial cable, the function $\varphi_{i}$ represents the voltage at node $i$. If all the 1D waveguides have the same length, $l$, it is easy to see from Eq. (1) that the network is periodic in $\omega$ with a period $2 \pi c / l$. Equation (1) is equivalent to a tightbinding Hamiltonian in an electronic system with a zero energy eigenvalue, i.e.,

$$
-\xi_{i} \varphi_{i}+\sum_{j} t_{i j} \varphi_{j}=0
$$

where $\xi_{i}=\Sigma_{j} \cot k l_{i j}$ and $t_{i j}=1 / \sin \left(k l_{i j}\right)$ are, respectively, the correlated-site energy and the hopping energy.

The band structures or dispersion relations $\omega(\boldsymbol{\kappa})$ of any periodic network can be obtained by solving a set of coupled equations in a unit cell with the use of the Floquet-Bloch theorem. As an example, we consider an infinite network with its nodes arranged in a square lattice. Only nearestneighbor nodes are connected by 1D waveguide of length $a$. 


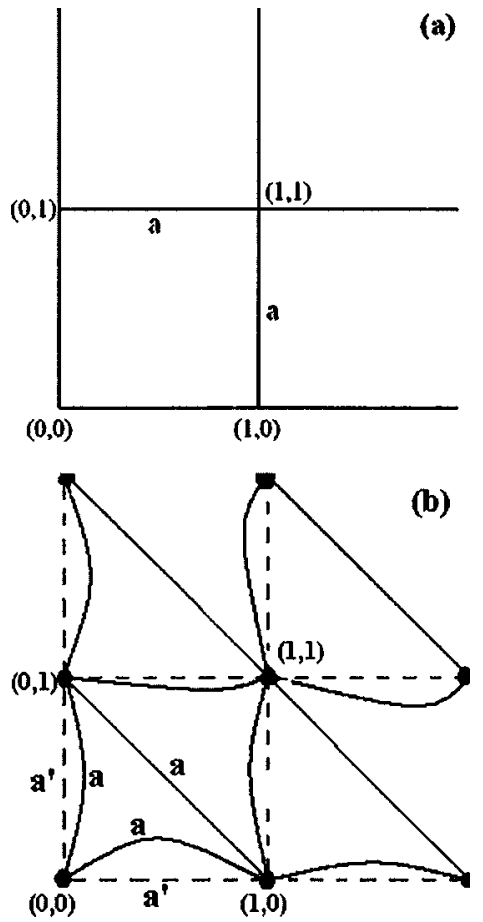

FIG. 1. The unit cell for (a) square network, (b) triangular network. All segments in the unit cells have the same length $a$. The lattice constants in (a) and (b) are, respectively, a and $a^{\prime}=a / \sqrt{2}$.

Figure 1(a) shows a unit cell with four nodes labeled by $(0,0),(1,0),(0,1)$, and $(1,1)$. The period of the unit cell is chosen to be $2 \mathrm{a}$ for convenience, which is twice as large as a conventional unit cell for a square lattice. The choice of a larger unit cell leads to a band folding effect and will not affect the structure of photonic band gaps in the system. From Eq. (1), we can write a set of networks equations for this unit as

$$
\begin{aligned}
& (-4 \cos k a) \varphi(0,0)+[\varphi(0,-1)+\varphi(0,1)+\varphi(1,0)+\varphi(-1,0)] \\
& \quad=0 \\
& (-4 \cos k a) \varphi(0,1)+[\varphi(-1,1)+\varphi(0,2)+\varphi(0,0)+\varphi(1,1)] \\
& \quad=0 \\
& (-4 \cos k a) \varphi(1,0)+[\varphi(1,-1)+\varphi(1,1)+\varphi(0,0)+\varphi(2,0)] \\
& \quad=0 \\
& (-4 \cos k a) \varphi(1,1)+[\varphi(2,1)+\varphi(1,2)+\varphi(1,0)+\varphi(0,1)] \\
& =0 .
\end{aligned}
$$

Because of the discrete translational symmetry in a periodic network, by using the Floquet-Bloch theorem, the wave functions at each node can be written as:

$$
\varphi_{\kappa_{x}, \kappa_{y}}(m, n)=e^{i \kappa_{x} m a} e^{i \kappa_{y} m a} U(m, n),
$$

where $\kappa_{x}$ and $\kappa_{y}$ are the reciprocal lattice vectors or the Bloch wave vectors in $x$ and $y$ direction, respectively. The indices $m$ and $n$ represent, respectively, the node indices in the $x$ and $y$ directions. $U(m, n)$ is a periodic function such that

$$
U(m+2, n+2)=U(m+2, n)=U(m, n+2)=U(m, n) .
$$

By using Eqs. (4) and (5), Eq. (3) becomes

$$
\left(\begin{array}{cccc}
-2 \cos k a & \cos \kappa_{x} a & \cos \kappa_{y} a & 0 \\
\cos \kappa_{x} a & -2 \cos k a & 0 & \cos \kappa_{y} a \\
\cos \kappa_{y} a & 0 & -2 \cos k a & \cos \kappa_{x} a \\
0 & \cos \kappa_{y} a & \cos \kappa_{x} a & -2 \cos k a
\end{array}\right) \cdot\left(\begin{array}{c}
U(0,0) \\
U(0,1) \\
U(1,0) \\
U(1,1)
\end{array}\right)=0
$$

where $k=\omega / c$. For each Bloch wave vector $\boldsymbol{\kappa}=\left(\kappa_{x}, \kappa_{y}\right)$ the corresponding eigenfrequencies $\omega(\boldsymbol{\kappa})$ are determined by the condition that the determinant in Eq. (6) vanishes, from which the band structure can be obtained.

The band structure of the square network is shown on the left-hand side of Fig. 2, where the symmetry points $\Gamma, X$, and $\mathrm{M}$ correspond to the wave vectors $\left(\kappa_{x}, \kappa_{y}\right)=(0,0),(\pi / 2 \mathrm{a}, 0)$, and $(\pi / 2 \mathrm{a}, \pi / 2 \mathrm{a})$, respectively. This figure shows that the square network does not possess a photonic band gap. It also shows that the band structures are periodic in $\omega$ with a period $\pi c / a$, which is half of the period shown in Eq. (1). This reason is given in the following. A square lattice is bipartite, i.e., it has two interpenetrating sublattices. For each eigenfrequency $\omega$ of a given $\boldsymbol{\kappa}=\left(\kappa_{x}, \kappa_{y}\right)$, we can always obtain another eigenstate with frequency $\omega+\pi c / a$ by changing the sign of all wave functions in one of the two sublattices. This can be seen easily from Eq. (6). If we make the following changes $k a \rightarrow k a+\pi, \quad U(0,0) \rightarrow-U(0,0)$, and $U(1,1) \rightarrow$ $-U(1,1)$, Eq. (6) remains unchanged. Such a reduction in the period does not apply to the triangular network as it is not bipartite.

To study the PBG structure in a triangular lattice, for convenience, we choose a unit cell that is plotted in Fig. 1(b), where one additional next-nearest-neighbor segment is inserted in each plaquette of a square lattice. However, this additional segment has the same length, $a$, as the nearestneighbor ones. Thus the segments on the four sides of the square have to be bended and its lattice constant becomes 
$a^{\prime}=a / \sqrt{2}$. The unit cell shown in Fig. 1(b) produces a lattice that is topologically equivalent to the triangular lattice. The symmetry points $\Gamma, \mathrm{X}, \mathrm{Y}$, and $\mathrm{M}$ correspond to the wave vectors $\left(\kappa_{x}, \kappa_{y}\right)=(0,0),\left(\pi / 2 \mathrm{a}^{\prime}, 0\right),\left(0, \pi / 2 \mathrm{a}^{\prime}\right)$, and $\left(\pi / 2 \mathrm{a}^{\prime}, \pi / 2 \mathrm{a}^{\prime}\right)$, respectively. Similar to the case of square lattice, we can use Eq. (4) to write a set of equations for the dispersion relations. The corresponding secular equation now takes the form

$$
\operatorname{det}\left(\begin{array}{cccc}
-3 \cos k a & \cos \kappa_{y} a^{\prime} & \cos \kappa_{x} a^{\prime} & \cos \left(\kappa_{x}-\kappa_{y}\right) a^{\prime} \\
\cos \kappa_{y} a^{\prime} & -3 \cos k a & \cos \left(\kappa_{x}-\kappa_{y}\right) a^{\prime} & \cos \kappa_{x} a^{\prime} \\
\cos \kappa_{x} a^{\prime} & \cos \left(\kappa_{x}-\kappa_{y}\right) a^{\prime} & -3 \cos k a & \cos \kappa_{y} a^{\prime} \\
\cos \left(\kappa_{x}-\kappa_{y}\right) a^{\prime} & \cos \kappa_{x} a^{\prime} & \cos \kappa_{y} a^{\prime} & -3 \cos k a^{\prime}
\end{array}\right)=0 .
$$

The band structure calculated from Eq. (7) is shown on the left-hand side of Fig. 3. A large PBG is found with a midgap frequency at $\omega_{c}=\pi c / a$. Unlike the case of the square network, the period of this band structure is $2 \pi c / a$ as dictated by Eq. (1) when all segments in a network have the same length, $a$.

The photonic band gap in a network system can also be studied by calculating the spectra of total transmission arising from a source inside the samples. This method is particularly useful for searching PBG when the structure has no translational symmetry (e.g., quasiperiodic or fractal networks, etc.). In this method, we inject wave energy from a particular node inside a finite-sized network and connect all boundary nodes by leads, which allow waves to leak out. For propagating states, the injected waves can propagate to the boundary nodes and give a finite total transmission. However, if the frequency is inside a PBG, the injected waves cannot propagate to the boundary node and, therefore, give a vanishing total transmission. Thus, a gap in the spectrum of total transmission is a signature of a PBG. Below we use a simple network to show how to calculate the total transmission arising from a given source.

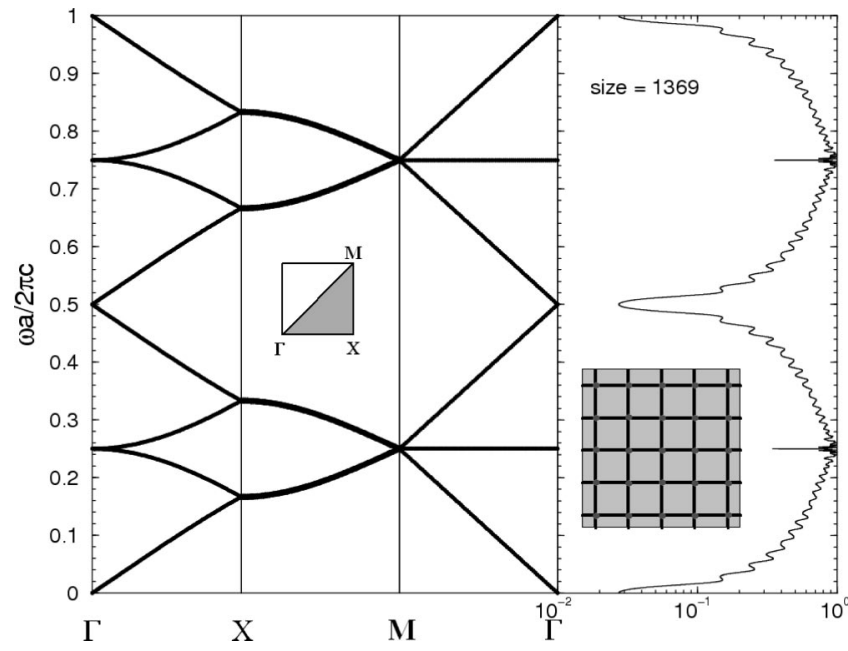

FIG. 2. Left panel: the band structure of the square network. Right panel: the total transmission of the square network with 1369 nodes. The shaded region of the inset on the left panel shows the Brillouin zone.
Consider an equilateral triangular loop connected by a lead at each node shown in Fig. 4. The network equations of this system have the following forms:

$$
\begin{aligned}
& \varphi_{0^{\prime}}-(3 \cos k a) \varphi_{0}+\varphi_{1}+\varphi_{2}=0 \\
& \varphi_{1^{\prime}}-(3 \cos k a) \varphi_{1}+\varphi_{2}+\varphi_{0}=0, \\
& \varphi_{2^{\prime}}-(3 \cos k a) \varphi_{2}+\varphi_{0}+\varphi_{1}=0,
\end{aligned}
$$

where $a$ is the length of each segment and $\varphi_{i^{\prime}}$ is the wave function in the lead at a distance $a$ away from the node $i$. If the waves are injected from node 0 and transmitted through nodes 1 and 2, the wave function in the lead at node 0 can be expressed as $\exp \left(i k s_{0}\right)+r \exp \left(-i k s_{0}\right)$, where $s_{0}$ has the origin at node 0 . Similarly, the wave functions in the leads at nodes 1 and 2 can be expressed as $t_{1} \exp \left(i k s_{1}\right)$ and $t_{2} \exp \left(i k s_{2}\right)$, respectively. Here, $r, t_{1}$, and $t_{2}$ are, respectively, the reflection and transmission amplitudes. Using these expressions, we

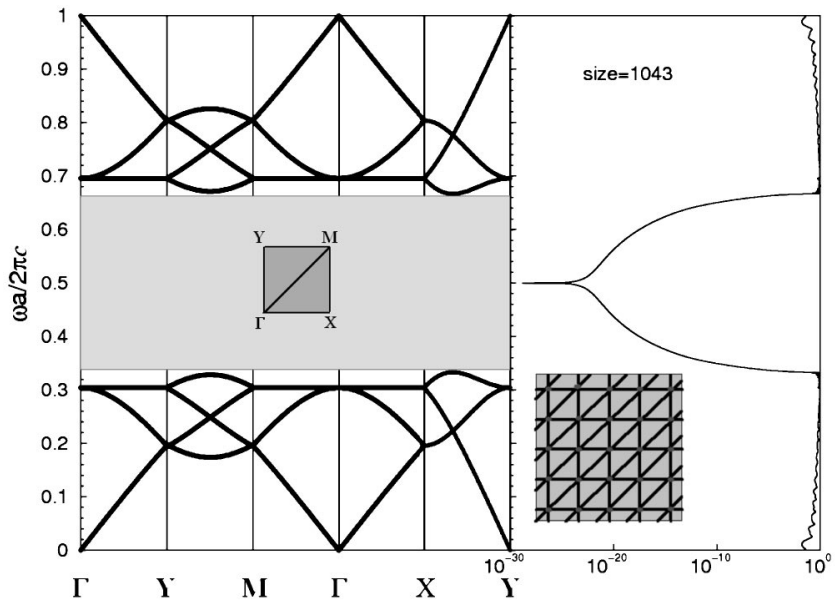

FIG. 3. Left panel: the band structure of the triangular network. Right panel: the total transmission of the triangular network with 1043 nodes. The shaded region of the inset on the left panel shows the Brillouin zone. 


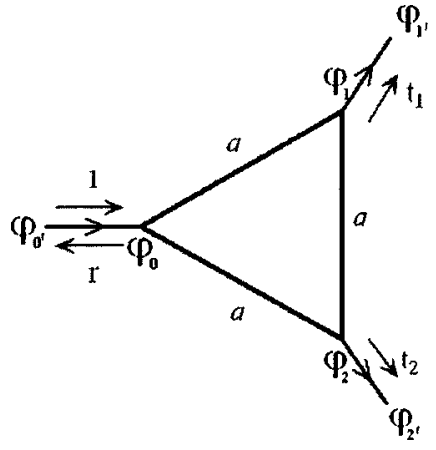

FIG. 4. A triangular loop with identical segment lengths, $a$. Leads are connected at the input and output nodes.

have $\varphi_{0^{\prime}}=\exp (-i k a)+r \exp (i k a), \varphi_{1^{\prime}}=t_{1} \exp (i k a)$, and $\varphi_{2^{\prime}}$ $=t_{2} \exp (i k a)$. By substituting the above expressions into Eq. (8), we solve the transmission problem of the network, from which we find the reflection and transmission coefficients as $R=|r|^{2}, T_{1}=\left|t_{1}\right|^{2}$, and $T_{2}=\left|t_{2}\right|^{2}$. The flux conservation requires that $R+T_{1}+T_{2}=1$. It should be noted that the reflection coefficient $R$ and the total transmission $T=T_{1}+T_{2}$ will not depend on the choice of positions $0^{\prime}, 1^{\prime}$, and $2^{\prime}$ in the leads, i.e., these positions can be arbitrary. Now we apply the above method to a triangular network containing 1043 nodes. The wave is injected from a node located at the center of the sample. Transmission leads are connected at each node on the boundary. The spectrum of total transmission is shown on the right-hand side of Fig. 3. A large gap is found in the spectrum of total transmission, which coincides with that of band structure calculation. Thus, both the band structure and total transmission show a large band gap with a midgap frequency at $\omega_{c}=\pi c / a$ and a width of $\Delta \omega=0.64 \omega_{c}$. The spectrum of total transmission for the square network is shown on the right-hand side panels of Fig. 2. No gap is found in this structure. This is also consistent with the band structure calculation. It should be noted that dips exist in the spectrum, but the total transmission at the dip is not exponentially

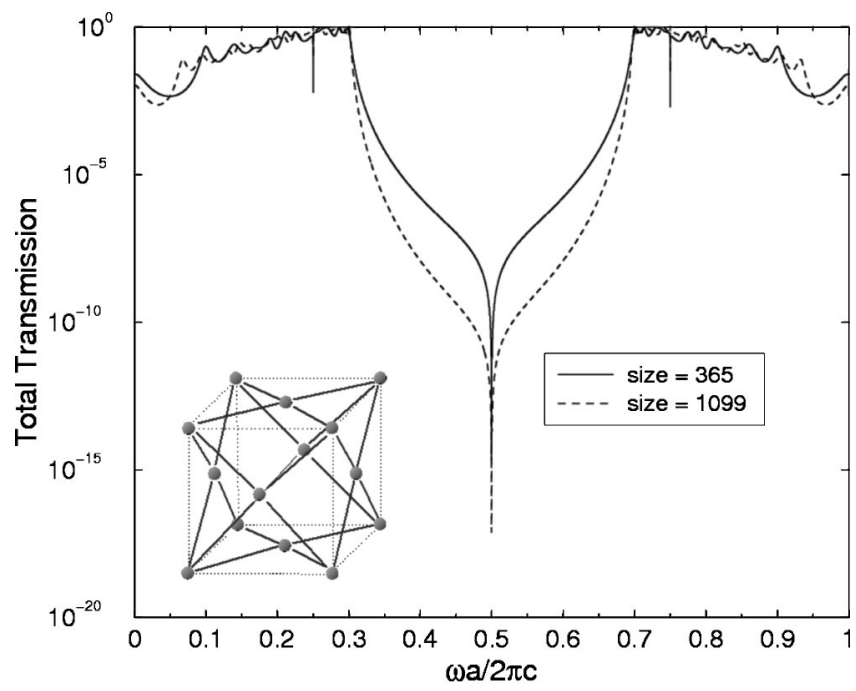

FIG. 5. The total transmissions of the face-centered cubic networks with 365 (solid line) and 1099 nodes (dash line).

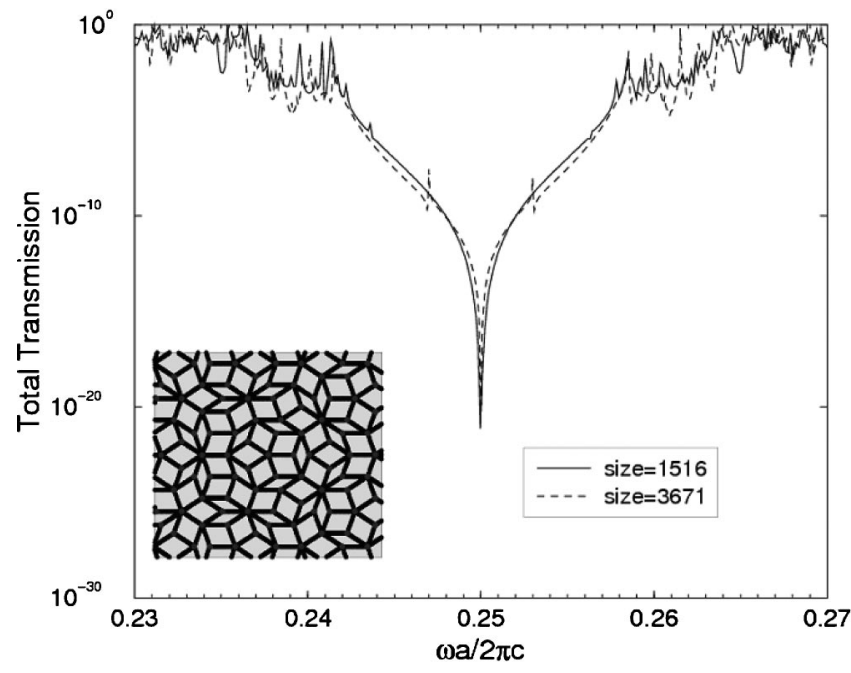

FIG. 6. The total transmissions of the pentagonal networks with 1517 (solid line) and 3671 nodes (dash line).

small compared to the gap in the triangular network in Fig. 3. The dip results from the small density of states around the corresponding frequency.

In general, it is more difficult to find large full gaps in three dimensions because its existence requires the pseudogaps in each direction to overlap with a large extent. For the network system, PBG can still exist in certain networks in three dimensions. The reason will be given in Sec. IV. Since the band structure of 3D networks is rather complicated, we present the total transmission instead. The total transmissions of a fcc network shown in Fig. 5 indicate that there is a large band gap with a midgap frequency $\omega_{c}$ $=\pi c / a$. The width of this band gap is $\Delta \omega=0.8 \omega_{c}$, which is $25 \%$ larger than that of the triangular network. In Fig. 5, the total transmissions of two sample sizes are plotted, where the solid line and dash line are, respectively, the total transmission of the fcc networks with 365 nodes and 1099 nodes. It is seen that gap position remains unchanged when the number of nodes is increased 3 times. However, the total transmission inside the gap of the larger sample is lower as expected.

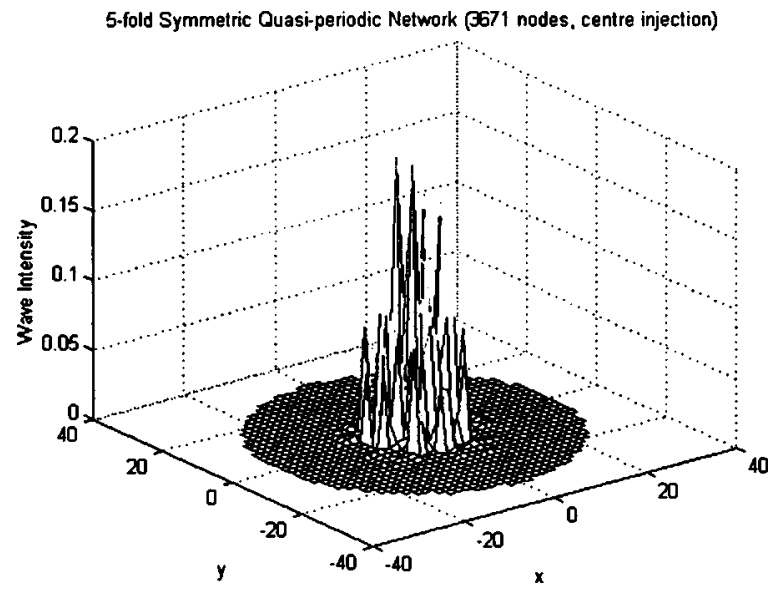

FIG. 7. The localized intensity distribution at frequency $\omega$ $=0.247(2 \pi c / a)$, for the pentagonal network with 3671 nodes. 


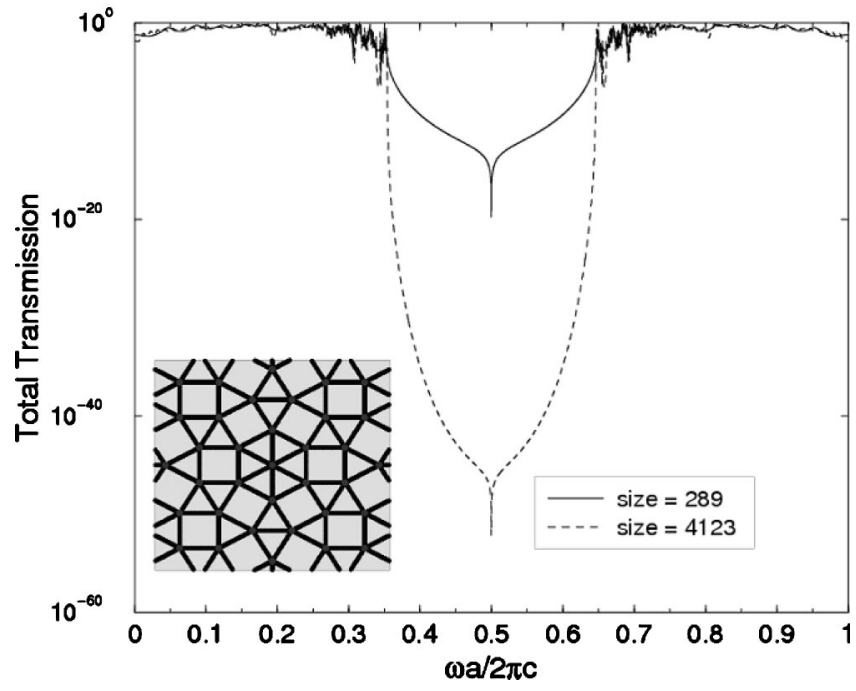

FIG. 8. The total transmissions of the dodecagonal networks with 289 (solid line) and 4123 nodes (dash line).

In addition to the periodic structures presented above, we have also studied the honeycomb network in 2D, the simple cubic, bcc, and diamond networks in 3D. However, no band gap is found in these structures.

\section{PBGs IN QUASIPERIODIC NETWORKS}

It is well known that quasiperiodic structures do not have spatial periodicity, and thus photonic band gaps that appear in a quasiperiodic structure may not have the same physical mechanism as in the case of a periodic lattice by Bragg diffraction. However, several references have reported PBGs in quasiperiodic structures. ${ }^{5-8}$ It was also suggested that a perfect long range periodic order may not be necessary to create a spectral gap in a dielectric $\mathrm{PBG}$ system provided that strong Mie-resonance scattering takes place at scattering centers. In the network system, we found that the existence of photonic band gap is quite insensitive to both the periodicity and dimensionality of the lattice, but is strongly correlated with the Mie-like scattering inside a single loop of waveguides. To illustrate this point, we have studied three kinds of quasiperiodic networks. Two of them are twodimensional networks and are, respectively, a pentagonal structure formed by Penrose tiling, with a fivefold symmetry, ${ }^{12,13}$ and a dodecagonal structure formed by square-triangle tiling, with a twelvefold symmetry. ${ }^{14}$ The third case is the three-dimensional network with icosahedral structure consisting of icosahedral symmetry that contains 15 twofold, 10 threefold, and 6 fivefold rotational axes. In this study, the icosahedral lattice is constructed by the sixdimensional hypercube projection method. ${ }^{15}$ The constructed structure has a node at the center of an icosahedron. The distance between the nodes on the faces and the node at the center of the icosahedron is given by

$$
\eta=\frac{\sqrt[4]{5} \sqrt{\tau}}{2} a=0.9511 a
$$

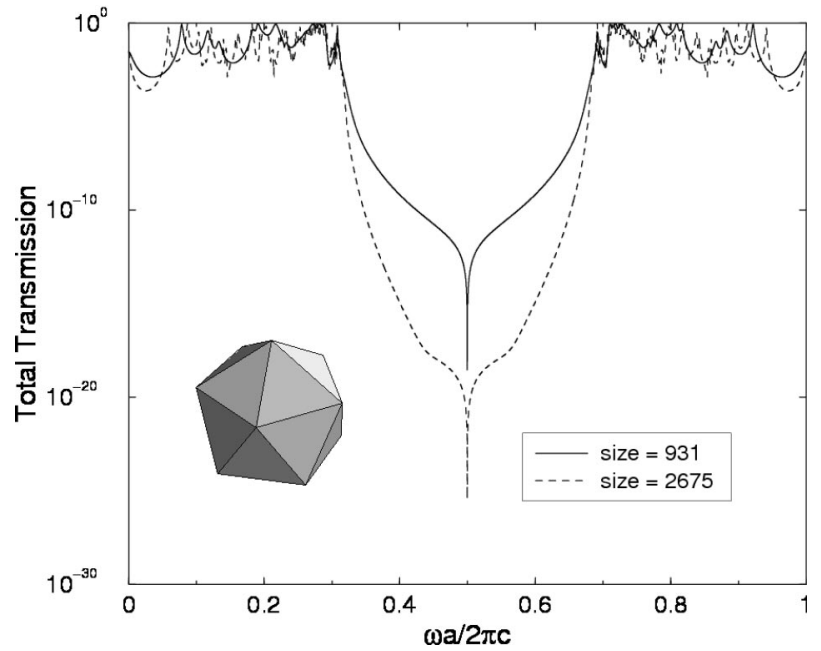

FIG. 9. The total transmissions of the icosahedral quasiperiodic networks with 931 (solid line) and 2675 nodes (dash line).

where $a$ is the distance between two nearby nodes at the faces and $\tau$ is the golden mean. For convenience, the nodes at the faces and the node at the center will also be considered as the nearest neighboring pairs, so that the largest coordination number of the icosahedral network is 12 .

We first consider the two-dimensional structures. The total transmissions of the pentagonal quasiperiodic networks, with 1516 (solid line) and 3671 nodes (dash line) are shown in Fig. 6. It is interesting to see that a large gap exists when the sample size is 1516 nodes. However, when the sample size is increased to 3671 nodes, new states appear inside the gap, which are characterized by the appearance of local peaks in the total transmission. These states are found to be localized states. For instance, the wave intensity profile of a localized state at the normalized frequency $\omega a / 2 \pi c=0.247$ is plotted in Fig. 7. Due to the appearance of localized states at large samples, it is possible that this gap would be continuously diminished when the size of the network is further increased. A different behavior is found in the dodecagonal quasiperiodic network. The total transmissions of the dodecagonal

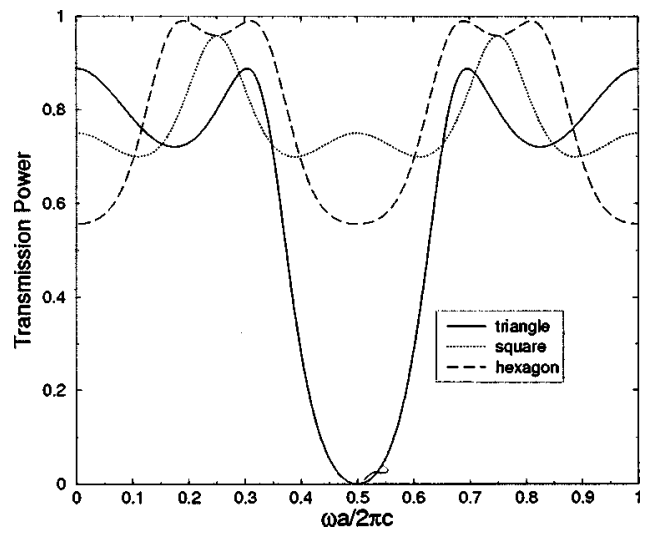

FIG. 10. The transmission spectra of the triangle (solid line), square (dotted line) and hexagon (dash line) loops, which are, respectively, the fundamental units in triangular, square, and honeycomb networks. 
TABLE I. The summary of photonic band gaps found in various networks. $\omega_{c}$ is the midgap frequency and $\Delta \omega$ is the width of the band gap.

\begin{tabular}{lcccc}
\hline \hline Networks & Dimensions & Periodicity & Fundamental building blocks & Band gap $\Delta \omega / \omega_{c}$ \\
\hline Square & 2 & Periodic & Rhombus & Nil \\
Triangular & 2 & Periodic & Triangle & 0.64 \\
Honeycomb & 2 & Periodic & Hexagon & Nil \\
Diamond & 3 & Periodic & Hexagon & Nil \\
Simple cubic & 3 & Periodic & Rhombus & Nil \\
bcc & 3 & Periodic & Rhombus & Nil \\
fcc & 3 & Periodic & Triangle, rhombus & 0.8 \\
Pentagonal & 2 & Quasiperiodic & Rhombus & Nil \\
Dodecagonal & 2 & Quasiperiodic & Triangle, rhombus & 0.6 \\
Icosahedral & 3 & Quasiperiodic & Triangle, rhombus & 0.78 \\
\hline \hline
\end{tabular}

networks with 289 (solid line) and 4123 nodes (dash line) are shown in Fig. 8. A large photonic band gap exists at $\omega_{c}$ $=\pi c / a$. The width of this band gap is $\Delta \omega=0.6 \omega_{c}$. This gap remains robust when the size of the network is increased from 289 to 4123 nodes. This suggests that a bona-fide large band gap can exist in the dodecagonal network.

In three dimensions, the icosahedral quasiperiodic network is also found to possess a large photonic band gap at the midgap frequency $\omega_{c}=\pi c / a$, according to the total transmission shown in Fig. 9. The number of nodes of the icosahedral networks are 931 (solid line) and 2675 (dash line). The width of the band gap is $\Delta \omega=0.78 \omega_{c}$, which is $30 \%$ larger than that of the dodecagonal quasiperiodic network, but is slightly smaller than that of the fcc network.

\section{DISCUSSION AND CONCLUSIONS}

It is remarkable that photonic band gaps exist in a number of network topologies without introducing any resonant loops. We summarize the intrinsic band gap properties of various periodic and quasiperiodic networks in Table I, where the fundamental building blocks and size/midgap ratio are listed and compared. In this table, it is obvious that only the networks that contain triangular loops in the fundamental building block possess photonic band gaps. This suggests that the existence of the large PBGs is related to the wave interference effect inside triangular loops in close analogy to the Mie scattering by spherical scattering centers. ${ }^{16}$ To confirm this observation, the transmission spectra of three loops with different connectivity are shown in Fig. 10. They are the triangular (solid line), square (dashed line), and hexagonal loops (dotted line), which are the fundamental units in triangular, square, and honeycomb networks. The transmission spectra are calculated by injecting waves to one of the leads and summing up the transmission coefficients at all others. From Fig. 10, it is found that although resonances appear in the square and hexagon loops, however, these resonances are not strong enough to produce a gap in any frequency in the respective networks. In addition to resonances, the triangular loop is capable of producing an antiresonance at frequency $\omega_{c}=\pi c / a$, where the total transmission is zero. This zerotransmission frequency is precisely the midgap frequency found in all the structures that possess gap in Table I. The full width at half-maximum of the transmission dip in the triangular loop is $0.5 \omega_{c}$. This is also roughly the size of the gaps shown in the table. It should be pointed out that antiresonance can exist only in ring-shape loops with odd number of nodes. The reason is as follows: For an even-node loop, an incident wave can propagate along two identical paths and interfere constructively at the node in the opposite side of the loop, thus, giving nonvanishing contribution to the total transmission.

In summary, we have investigated the propagation of waves in several network structures in 2D and 3D, including both periodic and quasiperiodic structures. Certain networks have large robust photonic band gap, namely the triangular, fcc, dodecagonal (12-fold), and icosahedral networks. The existence of such PBGs is found to be related to the existence of an antiresonance inside triangular loops of the fundamental building units. The gap positions in a network are also dictated by the frequencies at which the antiresonant transmission occurs. What we have studied here are intrinsic PBGs in network systems that do not have additional resonant loops. For those systems that do not have a gap, one can always create a gap by introducing additional resonant loops into the system as shown in Ref. 9. The gap size as well as its position can also be "tailor-made" by adjusting the resonant structure of the resonant loop.

\section{ACKNOWLEDGMENT}

This work was supported by Hong Kong RGC Grant No. 6112/98P. 
${ }^{1}$ J. D. Joannopoulos, R. D. Meade, and J. N. Winn, Photonic Crystals: Molding the Flow of Light (Princeton University Press, Princeton, 1995).

${ }^{2}$ Photonic Crystals and Light Localization, edited by C. M. Soukoulis (Kluwer Academic, Dordrecht, 2001).

${ }^{3}$ S. John, Phys. Rev. Lett. 58, 2486 (1987).

4 J. S. Foresi et al., Nature (London) 390, 143 (1997); R. De La Rue and C. Smith, ibid. 408, 653 (2000).

${ }^{5}$ Y. S. Chan, C. T. Chan, and Z. Y. Liu, Phys. Rev. Lett. 80, 956 (1998).

${ }^{6}$ S. S. M. Cheng, L. M. Li, C. T. Chan, and Z. Q. Zhang, Phys. Rev. B 59, 4091 (1999).

${ }^{7}$ M. E. Zoorob, M. D. B. Chariton, G. J. Parker, J. J. Baumberg, and M. C. Netti, Nature (London) 404, 740 (2000).
${ }^{8}$ X. Zhang, Z. Q. Zhang, and C. T. Chan, Phys. Rev. B 63, 081105(R) (2001).

${ }^{9}$ Z. Q. Zhang, C. C. Wong, K. K. Fung, Y. L. Ho, W. L. Chan, S. C. Kan, T. L. Chan, and N. Cheung, Phys. Rev. Lett. 81, 5540 (1998).

${ }^{10}$ Z. Q. Zhang and P. Sheng, Phys. Rev. B 49, 83 (1994).

${ }^{11}$ M. Li, Y. Liu, and Z. Q. Zhang, Phys. Rev. B 61, 16193 (2000).

${ }^{12}$ D. Levine and P. J. Steinhardt, Phys. Rev. B 34, 596 (1986).

${ }^{13}$ E. S. Socolar and P. J. Steinhardt, Phys. Rev. B 34, 617 (1986).

${ }^{14}$ M. Oxborrow and C. L. Henley, Phys. Rev. B 48, 6966 (1993).

${ }^{15}$ M. Mihalkovic, W.-J. Zhu, C. L. Henley, and M. Oxborrow, Phys. Rev. B 53, 9002 (1996).

${ }^{16}$ E. Lidorikis, M. M. Sigalas, E. N. Economou, and C. M. Soukoulis, Phys. Rev. Lett. 81, 1405 (1998). 